\title{
2011s-51
}

\section{Sustainable Network Dynamics}

Arnaud Z. Dragicevic, Bernard Sinclair-Desgagné

Série Scientifique
Scientific Series

\author{
Montréal \\ Juin 2011
}

(C) 2011 Arnaud Z. Dragicevic, Bernard Sinclair-Desgagné. Tous droits réservés. All rights reserved. Reproduction partielle permise avec citation du document source, incluant la notice (C)

Short sections may be quoted without explicit permission, if full credit, including () notice, is given to the source.

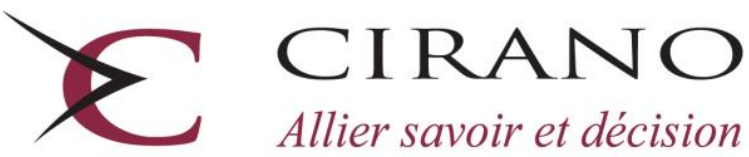

Centre interuniversitaire de recherche en analyse des organisations 


\section{CIRANO}

Le CIRANO est un organisme sans but lucratif constitué en vertu de la Loi des compagnies du Québec. Le financement de son infrastructure et de ses activités de recherche provient des cotisations de ses organisations-membres, d'une subvention d'infrastructure du Ministère du Développement économique et régional et de la Recherche, de même que des subventions et mandats obtenus par ses équipes de recherche.

CIRANO is a private non-profit organization incorporated under the Québec Companies Act. Its infrastructure and research activities are funded through fees paid by member organizations, an infrastructure grant from the Ministère du Développement économique et régional et de la Recherche, and grants and research mandates obtained by its research teams.

\section{Les partenaires du CIRANO}

\section{Partenaire majeur}

Ministère du Développement économique, de l'Innovation et de l'Exportation

\section{Partenaires corporatifs}

Autorité des marchés financiers

Banque de développement du Canada

Banque du Canada

Banque Laurentienne du Canada

Banque Nationale du Canada

Banque Royale du Canada

Banque Scotia

Bell Canada

BMO Groupe financier

Caisse de dépôt et placement du Québec

\section{CSST}

Fédération des caisses Desjardins du Québec

Financière Sun Life, Québec

Gaz Métro

Hydro-Québec

Industrie Canada

Investissements PSP

Ministère des Finances du Québec

Power Corporation du Canada

Rio Tinto Alcan

State Street Global Advisors

Transat A.T.

Ville de Montréal

\section{Partenaires universitaires}

École Polytechnique de Montréal

HEC Montréal

McGill University

Université Concordia

Université de Montréal

Université de Sherbrooke

Université du Québec

Université du Québec à Montréal

Université Laval

Le CIRANO collabore avec de nombreux centres et chaires de recherche universitaires dont on peut consulter la liste sur son site web.

Les cahiers de la série scientifique (CS) visent à rendre accessibles des résultats de recherche effectuée au CIRANO afin de susciter échanges et commentaires. Ces cahiers sont écrits dans le style des publications scientifiques. Les idées et les opinions émises sont sous l'unique responsabilité des auteurs et ne représentent pas nécessairement les positions du CIRANO ou de ses partenaires.

This paper presents research carried out at CIRANO and aims at encouraging discussion and comment. The observations and viewpoints expressed are the sole responsibility of the authors. They do not necessarily represent positions of CIRANO or its partners. 


\title{
Sustainable Network Dynamics*
}

\author{
Arnaud Z. Dragicevic ${ }^{\dagger}$, Bernard Sinclair-Desgagnét
}

\begin{abstract}
Résumé / Abstract
Nous proposons un modèle dynamique de gestion des écosystèmes par la théorie des graphes en tant que contrôle d'un système en réseau composé de nœuds cibles et de nœuds non identifiés. Le réseau est représenté par un graphe complet dans lequel tous les nœuds sont connectés par une arête unique. Les nœuds cibles sont attirés par une fonction objectif issue d'un processus externe de gestion des écosystèmes. Ils tirent le réseau vers la position de l'objectif qui peut être non-nulle ou stationnaire. La politique de gestion est considérée réussie si le graphe reste connecté dans le temps, c'est-à-dire que les nœuds cibles atteignent l'objectif et les nœuds non identifiés restent dans l'enveloppe convexe. Lors de la transposition du réseau écosystémique dans le temps, le modèle génère un Théorème de l'Impossibilité ainsi qu'un Critère de Durabilité qui maintient la pleine connectivité du réseau. Ce dernier peut aisément être relié à la définition générale de la durabilité comme la préservation de l'intégrité écologique. Enfin, nous identifions trois règles de gestion pour assurer le maintien de la connectivité dans le temps, sachant les propriétés de la fonction objectif de transposition, la nature des connexions, et les retards de réactualisation de l'utilité entre les nœuds.
\end{abstract}

Mots clés : bioéconomie, gestion des écosystèmes, théorie des graphes, connectivité.

We propose a dynamic graph-theoretic model for ecosystem management as a control over networked system composed of target nodes and unmarked nodes. The network is represented by a complete graph, in which all vertices are connected by a unique edge. Target nodes are attracted by the objective function issued from the external ecosystem management. They pull the network towards the objective position, which is either non-null or stationary. The management policy is considered successful if the graph remains connected in time, that is, target nodes attain the objective and unmarked nodes stay in the convex hull. At the time of the ecosystem network transfer, the model yields an Impossibility Theorem as well as a Sustainability Criterion to maintain full connectivity of the network. The latter can be easily linked to the general definition of sustainability as ecosystem integrity preservation. At last, we identify three management rules to ensure the maintenance of connectivity in time, given the properties of the objective transposition function, the nature of connections and utility updating time-delays between the nodes.

Keywords: bioeconomics, ecosystem management, graph theory, connectedness.

Codes JEL : Q2, Q5

\footnotetext{
${ }^{*}$ We would like to thank John Galbraith for his valuable comments. The usual caveats apply.

† CIRANO, arnaud.dragicevic@cirano.qc.ca.

${ }^{\ddagger}$ HEC Montréal and Ecole Polytechnique ParisTech.
} 


\section{Introduction}

"Ecosystem management is management driven by explicit goals, executed by policies, protocols, and practices, and made adaptable by monitoring and research based on our best understanding of the ecological interactions and processes necessary to sustain ecosystem composition, structure, and function" (Christensen et al. 1996).

Ecosystem management aims at maintaining ecosystem health and integrity, given the numerous goods and services they provide. Health is the capacity of ecosystems to maintain their functions (Constanza et al. 1992). We can mention hydrologic flux and storage, biological productivity, maintenance of biological diversity as some of the ecosystem processes; food, construction materials, medicinal plants and tourism as part of the ecosystem goods; maintenance of hydrological cycles, climate regulation, sanitization of air and water, maintenance of the gaseous composition, etc. as some of the ecosystem services. In a word: they are vital for all forms of life. Integrity consists in maintaining ecosystem's selforganizing structural complexity (Callicott 1993).

Ecological systems are dynamic systems because their change is normal and ubiquitous, so sustainability does not imply maintenance of the status quo. Besides, ecosystem status quo usually leads to failure in the long term (Connell and Sousa 1983). Studies of ecosystems as evolutionary networked systems have recently been proposed (Strogatz 2001, Fath 2004, Jordan and Scheuring 2004, Fath and Grant 2007, Lanzen 2007) but these studies do not consider connectivity issues. From the ecological perspective, high connectivity implies much interaction of animals, plants, energy, water, nutrients or other matter among elements (Cantwell and Forman 1993).

The behavior of the ecosystem depends on the exchange of conservative resources between organisms. The network analysis enables to represent these organisms and exchanges 
as a collection of storages and flows (Fath and Patten 1998). The importance of interconnections within ecosystems is one of the most important lessons learned from ecological research and natural resource management experience (Peterson 1993). Ecosystems are connected networks (Patten 1984, Higashi and Patten 1989). They are regulated across many control variables in interactive networks. Pichai et al. (2001) developed graph-theoretic procedures to identify the minimal sets of connections which are essential for preserving the structural properties of the system. Because a precise number of species to maintain key ecosystem processes is futile (Christensen et al. 1996), we do not reduce ecosystem networks to their minimal sets and study their general connectivity instead. We identify network targets under the strict constraint of complete connectedness maintenance. No formal model about the connectivity issue for the networked system control in the ecosystem management has yet been proposed. This paper is a response to this shortage.

Our work was inspired by the literature on controllability and stability of leaderfollower networks (Ji et al. 2008, Rahmani et al. 2009, Gustavi et al. 2010). We consider the ecosystem management as a control over networked system composed of target nodes and unmarked nodes. Target nodes can be biodiversity elements that need to be managed in order to achieve the environmental policy, and unmarked nodes are then all the other elements indirectly incorporated in the policy, for they are fully connected to target nodes. The network is represented by a complete graph, in which all vertices are connected by a unique edge. Target nodes are attracted by the objective function issued from the external human ecosystem management. They pull the network towards the objective position. The objective can either be reconfiguration or stationarity upholding. On the one hand, the objective function, which depends on the distance between the objective position and the target node position, can be non-null, implying that target nodes must reach new positions set up by the 
environmental policy. On the other hand, the objective function can be null or stationary. In this case, target nodes must be maintained as they stand. The environmental policy then seeks to avoid degradation or deterioration of targeted elements, which would cause the ecosystem vulnerability. The management policy is considered successful if the graph remains connected in time, that is, target nodes attain the objective and unmarked nodes remain within the graph.

Fisher et al. (1991) call for coordinated controls in a dynamic model of fishery and water resource management. Albers (1996) presents a spatial-intertemporal model for economic management of tropical forests. She introduces ecological constraints such as spatial interactions. However, her focus is on impact of the uncertainty and the discount rate. Smith et al. (2009) consider both linked subsets and distributed controls, but they use estimating models of parameters subjected to optimization techniques. Our will is to apprehend a general sustainable management towards natural systems, when the latter are viewed as dynamic networks, but also to display policy rules for an efficient management.

We propose a dynamic graph-theoretic model, in which the evolution of the network given the objective function is studied. It yields a theorem of impossibility within the ecosystem network transfer as well as a sustainability criterion to maintain full connectivity of the network in time, which enables to safeguard its utility domain. Our definition of sustainability can be easily linked to the general definition of sustainability as the ecosystem integrity preservation. Furthermore, our model can stand for wanting theoretic background needed for decision support systems in ecosystem management (Reynolds 2005, Jensen et al. 2009). Although theoretic models are often criticized because they simplify the factual complexity, they can be highly useful in identifying sensitive ecosystem components or in simulating alternatives (Lee 1993).

For example, the inability to monitor and manage all aspects of biodiversity has led to the development of paradigms that focus either on single species or whole ecosystems. Both 
have advocates and detractors (Payton et al. 2002). The keystone species concept can allow managers to combine the best features of both paradigms. A keynote species is considered a species whose effect is disproportionally large relative to its abundance (Power et al. 1996). Our model encompasses both paradigms in a sense that targeting specific nodes, which can be interpreted as keynote species among other things, does not neglect the complexity and high connectedness of the entire ecological network.

After this starting section, we introduce the ecosystem management model in Section 2. Section 3 clarifies the methodology of subset identification. The dynamic behavior of network is modeled in Section 4. Ensuing management prescriptions are given in Section 5. Section 6 concludes.

\section{The model}

We consider network closed-loop systems in which the system outputs are used as the system inputs. We know that an ecosystem component has a dual role as a receiver and transmitter of interactions (Patten 1981). Let $N$ be the number of nodes evolving in $\mathbb{R}^{2}$. The two dimensional case corresponds to nodes moving either towards the objective position or maintaining themselves at a certain distance with their surroundings. Let $x_{i} \in \mathbb{R}^{2}$ denote the position of node $i$ in the network. The set of all possible positions of the dynamical system is the configuration space. It is spanned by the stack vector of all the control inputs $x=\left[x_{1}^{T}, \ldots, x_{N}^{T}\right]^{T}$ which denotes the aggregated state of the network as an involution. In our case, involution implies the maintenance of the network during the dynamic transposition to an objective. The stack vector reflects the environmental policy space towards the network, for the control inputs are amassed and configured so as to arrive at the environmental objectives. 
The trajectory of a node obeys the model of a single-integrator dynamics

$$
\dot{x}=c_{i}, i \in \Lambda=\{1, \ldots, N\}
$$

where $c_{i}$ denotes the control input for each node. The ecosystem manager identifies the subset of target nodes $\Lambda^{t}$ and the subset of unmarked nodes $\Lambda^{u}$, such that

$$
\Lambda^{t} \cup \Lambda^{u}=\Lambda \text { and } \Lambda^{t} \cap \Lambda^{u}=\varnothing
$$

The number of nodes in each subset is given by $\left|\Lambda^{t}\right|=N_{t}$ and $\left|\Lambda^{u}\right|=N_{u}$ respectively. Each node has a utility domain $u \in(0, \Delta]$, from which it gets utility from other nodes. This utility domain depends on the Euclidean distance $d$ from other nodes. All nodes within the utility domain of a node form its utility node set.

Definition 1: For $i, j \in \Lambda$, and for node i's utility node set $\Lambda_{i}=\left\{j \in \Lambda_{i}: 0<\left|x_{i}-x_{j}\right| \leq \Delta\right\}$, the utility domain $u_{i j}$ is defined as $u_{i j}=\mathrm{I}_{\Lambda_{i}}\left(d_{i j}\right)$ where

$$
\mathrm{I}_{\Lambda_{i}}\left(d_{i j}\right)= \begin{cases}d_{i j} & \text { if } j \in \Lambda_{i} \\ 0 & \text { if } j \notin \Lambda_{i}\end{cases}
$$

Definition 2: For $\forall j \in \Lambda$, the network utility domain $U_{i}(\Lambda)=\sum_{j} u_{i j}$ is the sum of utility domains contained in the network utility set. 
Interactions in the system are represented by a complete graph $\Gamma=\{V, E\}$ which defines the topology. The graph consists of vertices $V=\{1, \ldots, N\}$ indexed by the node members and the set of edges $E=\left\{(i, j) \in V \times V \mid i \in \mathrm{N}_{j}\right\}$ that represent inter-node interactions. The set of edges $E$ and the graph $\Gamma$ vary in time.

The Euclidean norm distance between two random nodes $i$ and $j$ is

$$
d_{i j}=d_{j i}=\left|x_{i}-x_{j}\right|=\left[\left(x_{i}-x_{j}\right)^{T}\left(x_{i}-x_{j}\right)\right]^{\frac{1}{2}} \geq 0
$$

So the time derivative of the squared distance equals

$$
\dot{d}_{i j}^{2}=2 d_{i j} \dot{d}_{i j}=2\left(x_{i}-x_{j}\right)^{T}\left(\dot{x}_{i}-\dot{x}_{j}\right)
$$

When the distance equals the utility domain or $d_{i j}=u_{i j}$, the condition for nodes $i$ and $j$ to evolve connected is $\dot{d}_{i j}^{2} \leq 0 . \dot{d}_{i j}$ depends on the trajectories of nodes $i$ and $j$.

\section{Subsets identification}

We have ecosystem networks represented by graphs composed of a large number of nodes. Ecosystem managers can either select a group of target nodes or identify a subset of target nodes by linking nodes according to their interactions. In the second case, how can we identify the subsets of target nodes? We have 3 types of interactions between nodes:

- $\theta_{t \mid t}:$ Target node-target node type of interaction

- $\theta_{u \backslash u}:$ Unmarked node-unmarked node type of interaction

- $\quad \theta_{t \mid u}:$ Target node-unmarked node type of interaction 
Let us select a node from the graph and start to connect it to other nodes by any of the three types of interactions. By choosing the type of interaction, we implicitly identify target and unmarked nodes and their respective subsets. This method brings us to the rationale of Ramsey's coloring of the edges in a graph in more than one color (Graham et al. 1990, Chomette 2010).

Let $n$ denote a large number of nodes in a complete graph $\Gamma_{n}$. Let $k$ be the number of nodes in subgraph $\Lambda_{\theta_{i}}^{k} \subseteq \Gamma_{n}$ in which all nodes are connected by the same type de interaction $\theta_{i}$ (for $i \leq r$ ). We call $\Lambda_{\theta_{r}}^{k}$ a monotype subgraph of $k$ nodes.

We note $n \rightarrow\left(k_{\theta_{1}}, k_{\theta_{2}}, \ldots, k_{\theta_{r}}\right)$ if $n$ is such that connecting nodes in $\Gamma_{n}$ induces a monotype subgraph $\Lambda_{\theta_{r}}^{k}$ in which all interactions are of $\left(\theta_{i}\right)_{i \leq r}$ th type. This means that $n$ nodes in $\Gamma_{n}$ are contained in $\Lambda_{\theta_{r}}^{k}$ subgraphs of $k$ nodes. In this monotype subgraph $\Lambda_{\theta_{r}}^{k}$, all nodes are connected either by $\theta_{t \backslash t}$ or by $\theta_{u \backslash u}$ or by $\theta_{t \backslash u}$. When $k_{\theta_{1}}=k_{\theta_{2}}=\ldots=k_{\theta_{r}}$, we have $n \rightarrow(k)_{\theta_{r}}$. In our case, we need to consider $r=3$.

Identification Theorem (Ramsey): Let $k$ and $r$ be integers. For every interaction in $\Gamma_{n}$ in one of $\left(\theta_{i}\right)_{i \leq r}$ types, there exists $n$ such that $\Gamma_{n} \supseteq \Lambda_{\theta_{r}}^{k}$.

Proof in the Appendix.

We now know that the subset of target nodes exists. It then belongs to the ecosystem manager to decide on the 'coloring' of the ecosystem network. While in Ramsey's work, the coloring of edges did not imply anything else but the number of colors, we have different types of interactions which decide on specific attributes of the nodes at stake. Indeed, if the ecosystem 
manager decides to connect two nodes by $\theta_{t \mid t}$, she specifies that both nodes are target nodes. The same reasoning applies with other two types of interactions. Therefore, some limitations on the coloring must be posited in order to achieve the identification of the subgraphs.

Coherence Principle: Let $i$ and $j$ be two connected vertices in the graph such that $\left\{(i, j) \in V \times V \mid i \in \Lambda_{\theta_{i}}\right\}$ and $i \in \Lambda^{\theta_{i}}$. For any vertex $k$, one must verify $\left\{(i, k) \in V \times V \mid i \in \Lambda_{\theta_{i}}\right\}$.

The Principle says that adding an extra node to a subset of connected nodes must not modify the attributes of already connected nodes. Otherwise, the paradox of multiple attributes of one node can occur, making the subsets of target and unmarked nodes unidentifiable.

In terms of graph construction, it means that the starting interaction of two identified nodes within the graph decides on the respective attributes of those nodes. All the remaining interactions in the network must be made from this starting subset and each additional interaction in the graph must be decided with respect to the existing attribute of already connected nodes.

\section{Dynamic Network Behavior}

The dynamics of unmarked nodes is given by the Laplacian-based control strategy (consensus) differential equation, meaning that each node moves in the direction of the average position of its utility nodes (Mesbahi and Egerstedt 2010). Indeed, the interaction between nodes' dynamics is realized through the control input $c_{i}(t)$, assumed to be the sum of the differences between states of a node and its neighbors. We introduce the utility updating time-delay parameter $\varepsilon \in[0,1]$, such that $\varepsilon=1$ means the absence of delay. When the node is transposed to some coordinates issued from the ecosystem management, it might update its 
utility from its utility nodes with a certain amount of delay. Time-delay can be problematic should the nodes update their utility coordinates too tardily to remain connected to other nodes; the rationale is that the time-delay can jeopardize the connectivity between the nodes. Our way of modeling time-delay differs from that of Olfati Saber and Murray (2004) but keeps the aim of studying consensus criteria when update responses are delayed.

For a random unmarked node $i \in \Lambda^{u}$ we have

$$
\dot{x}_{i}=-N_{i} x_{i}+\varepsilon_{i} \sum_{k \in \Lambda_{i}} x_{k} \quad \forall i \in \Lambda^{u}
$$

This dynamics is completely distributed. Indeed, a node needs only to obtain the utility of its utility nodes in order to implement its own utility update. A consensus problem is when spatially distributed elements of a network must reach overall utility without recourse to a central coordinator (Spanos et al. 2005). As well, the Laplacian dynamics has the advantage of converging to a steady-state, and we know that natural ecosystems operate near steadystates (Patten 2010).

The dynamics of target nodes is also based on the consensus equation but also on the objective term which transposes the network to the objective position $x=g$. For node $i$ assume $d_{i}=\left|g-x_{i}\right|$. The dynamics for a random target node $i \in \Lambda^{t}$ is given by

$$
\dot{x}_{i}=-N_{i} x_{i}+\varepsilon_{i} \sum_{k \in \Lambda_{i}} x_{k}+F\left(x_{i}, g\right) \forall i \in \Lambda^{t}
$$

where $F\left(x_{i}, g\right)$ is the objective transposition function such as 


$$
F\left(x_{i}, g\right)= \begin{cases}f\left(\left|g-x_{i}\right|\right)\left(g-x_{i}\right) & \text { if } d_{i}>0 \\ 0 & \text { if } d_{i}=0\end{cases}
$$

At any $x_{i} \neq g$, the direction of $F\left(x_{i}, g\right)$ is towards the objective position and the magnitude is decided by a continuous scalar function $f\left(d_{i}\right) \geq 0$ which depends on node $i$ 's distance to $g$. $F\left(x_{i}, g\right)$ is continuous when $f(0)=0$ and $\lim _{x \rightarrow g} F(x, g)=0$. The objective transposition function comes from the attraction-repulsion functions used in swarm (Gazi and Passino 2004) and rendezvous (Gustavi et al. 2010) models. In these models, the motion dynamics also depends on the distance between two agents and the function of attraction $f(\cdot)$ without the recourse of a central coordinator. In a swarm model considered in a Euclidean space, all agents move simultaneously and know the exact relative position of all other agents.

For $i \in \Lambda^{u}$ the trajectory is given by

$$
\dot{x}_{i}=-N_{i} x_{i}+\varepsilon_{i} \sum_{k \in \Lambda_{i}} x_{k}
$$

If $N_{i}=0$, the unmarked node will not move. When the graph stays connected, the consensus equation drives nodes to the same state value. Thus, if $N_{i}>0$, by setting $\alpha=\frac{1}{N_{i}}$, we obtain

$$
\alpha \dot{x}_{i}=-x_{i}+\varepsilon_{i} \sum_{k \in \Lambda_{i}} \frac{x_{k}}{N_{i}}
$$

Corollary 1: The trajectory of unmarked node converges to the barycenter of the subgraph $\Lambda_{i} \subseteq \Gamma$

For $i \in \Lambda^{t}$, we have 


$$
\dot{x}_{i}=-N_{i} x_{i}+\varepsilon_{i} \sum_{k \in \Lambda_{i}} x_{k}+f(|g-x|)\left(g-x_{i}\right) .
$$

If $N_{i}=0$, the target node will, depending on $f(|g-x|)$, either not move or head to $g$. If $N_{i}>0$ and $F\left(x_{i}, g\right) \neq 0$, by setting $\beta=\frac{1}{N_{i}+f(|g-x|)}$ we get

$$
\beta \dot{x}_{i}=-x_{i}+\frac{N_{i} \varepsilon_{i} \sum_{k \in \Lambda_{i}} \frac{x_{k}}{N_{i}}+f\left(\left|g-x_{i}\right|\right) g}{N_{i}+f\left(\left|g-x_{i}\right|\right)} .
$$

Corollary 2: The trajectory of target node converges to an aggregate of the barycenter of the subgraph $\Lambda_{i} \subseteq \Gamma$ and the objective position $g$.

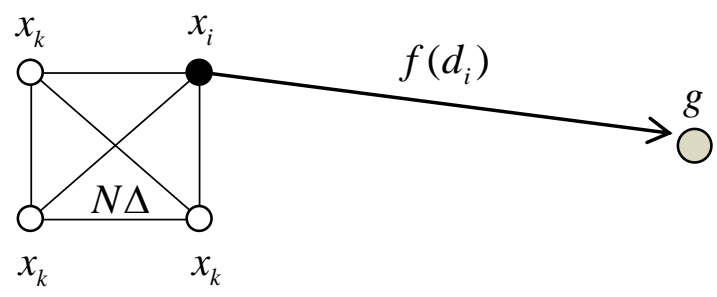

Fig. 1 Complete graph with an objective position

(9) and (11) guarantee the boundedness of solutions of the system. This property yields the following lemma.

Lemma 1: Let $\Gamma$ be a graph consisting of unmarked and target nodes. When $\Omega$ is the convex hull containing the nodes in $\Gamma$ and the objective position $g$, we have

$$
\Omega=\operatorname{Co}(\Gamma \cup g)
$$


Proof (see Ji et al. 2008 and Gustavi et al. 2010)

For $i \in \Lambda^{u}$, if $N_{i}>0$, the trajectory of unmarked node converges to the barycenter of the subgraph $\Lambda_{i} \subseteq \Gamma$. Due to convexity, the barycenter lies within the convex hull. We conclude that the trajectory of unmarked node $i$ lies within $\Omega$.

For $i \in \Lambda^{t}$, if $N_{i}>0$, the trajectory of $i$ converges to an aggregate of the barycenter of the subgraph $\Lambda_{i} \subseteq \Gamma$ and the objective position $g$. By the convexity of $\Omega$, node $i$ remains in $\Omega$.

Since the trajectories of both the unmarked and target nodes are on the boundary or in the interior of $\Omega$, both nodes stay inside the convex hull. $\Omega_{0}$ is thus an invariant set.

At the time interval $t=0$, the convex hull is $\Omega_{0}$. Now assume that none of the nodes are connected to any utility nodes $x_{k}, k \notin \Gamma$, and that $f(|g-x|) \leq f_{\max }\left(d_{j}\right)<\infty \quad \forall x \in \Omega_{0}$. $f_{\max }\left(d_{j}\right)$ is the largest value of $f\left(d_{j}\right)$ in the convex hull. As the time interval $t \rightarrow \infty$, the trajectories of nodes in $\Gamma$ evolve within $\Omega_{0}$. Although the dynamics of target and unmarked nodes do not need to be identical, the objective of the ecosystem manager is to bring the network to a certain position. Whenever $d_{i}>0$, given that $\lim _{t \rightarrow \infty} x_{i}(t)=g \forall i \in \Lambda$ we have

$$
\beta \dot{x}_{i}=\alpha \dot{x}_{i}
$$

which yields 


$$
g=\varepsilon_{i} \sum_{k \in \Lambda_{i}} \frac{x_{k}}{N_{i}}
$$

For $F\left(x_{i}, g\right) \neq 0$, the barycenter of the subgraph $\Lambda_{i} \subseteq \Gamma$ converges (with the risk of delay) to the objective position $g \forall i \in \Lambda$. Therefore, in the limit, the objective position and the barycenter overlap. This is impossible because of Corollaries 1 and 2. Pragmatically, this means that the ecosystem manager cannot bring the network center of gravity to the objective position. The following theorem ensues.

Impossibility Theorem: As $t \rightarrow \infty$ the terminal barycenter of the subgraph $\Lambda_{i} \subseteq \Gamma$ cannot be $g$ itself; it can at most be near the objective position within the convex hull $\Omega$.

Proof

By Corollaries 1 and 2, we know that the trajectories of unmarked nodes follow the trajectories of target nodes which converge to an aggregate of the barycenter and $g$. By (13), we know that the barycenter of the subgraph $\Lambda_{i} \subseteq \Gamma$ attains the objective position $g \forall i \in \Lambda$, a result that contradicts the precedent. Finally, by Lemma 1, we know that $\Omega=\operatorname{Co}(\Gamma \cup g)$. This ends the proof.

\section{Management prescriptions}

The only constraint that can be imposed upon the transposition of the network near the objective position is that the graph remains connected. This brings us to the following criterion. 
Sustainability Criterion: For $\dot{x}$, assume $x(0) \in \Omega$. If Lemma 1 holds and $\lim _{t \rightarrow \infty} x_{i}(t)=g$ $\forall i \in \Lambda$, the graph $\Gamma(t)$ remains connected.

Proof (see Dattorro 2005 and Gustavi et al. 2010)

By setting the dynamics via the matrix Kronecker product in stack vector form of the node's positions, we have

$$
\dot{x}=-\left[\left(L \otimes I_{2}\right)\left[x_{1}, \ldots, x_{N}\right]^{T}+\left[F_{1}(x, g), \ldots, F_{n}(x, g)\right]^{T}\right]
$$

with $L$ the Laplacian matrix of $\Gamma(t)$ and $I$ the identity matrix of size 2 . Let $g=[g, \ldots, g]^{T}$. Taking $V=\frac{1}{2}(x-g)^{T}(x-g)$ as a graph-compatible Lyapunov function, which can be considered as a function of time, the time derivative yields

$$
\dot{V}=-\left[(x-g)^{T}\left(L \otimes I_{2}\right)(x-g)\right]-\sum_{i \in \Lambda^{t}} F\left(x_{i}, g\right)
$$

where $V \leq 0$ is negative semi-definite due to the eigen-properties of $L=L^{T}$, which is symmetric positive semi-definite, and $-I$ and $F$ being monotically increasing. As long as the graph is connected, then $L$ is positive semi-definite and, as such, $-L$ is negative semidefinite. LaSalle's invariance principle addresses the asymptotic stability of a system. Given the convergence to the objective, the principle gives $x-g \rightarrow 0 \quad \forall i \in \Lambda^{t}$ as $t \rightarrow \infty$ and $(x-g)^{T}\left(L \otimes I_{2}\right)(x-g) \rightarrow 0$. The system is then stable and $x-g$ will tend to the null-space of $L$ asymptotically. This implies that all scalar positions of all the nodes will tend to the 
same value. For any vector with identical components such as $x=g$, any consensus is an equilibrium. Therefore, the dynamics must converge to a steady state.

Since $g$ is in $\Omega$ and $\Omega$ is convex, $i$ remains in $\Omega$. By Lemma 1, we know that $\Omega$ is an invariant set, and hence the connectivity is ensured. This concludes the proof.

$\lim _{x \rightarrow \infty} x_{i}(t)=g \quad \forall i \in \Lambda$ explains that the trajectory of a node, be it a target node or an unmarked node, can at most attain $g$. Given the Impossibility Theorem, the condition reveals that the nodes remain at best within $\Omega$, as the barycenter cannot overlap with $g$ in $\Gamma(t)$.

Sustainability Criterion is the minimal constraint which enables a network to reach the objective without compromising its connectivity. Put differently, the condition is a security that the graph transposes to the objective position without disconnecting, because disconnections induce vulnerability of the system. Further, provided that the graph remains connected, any Laplacian consensus is an equilibrium.

Nodes are initially within the utility domain, that is, at a distance $d_{i j}<\Delta$. Hence, the initial graph $\Gamma(0)$ is connected. For an ordered set $W=\left\{x_{1}, x_{2}, \ldots, x_{k}\right\} \subseteq V$ and a vertex $x_{i}$ of $\Gamma$, we set up the metric representation of $x_{i}$ with respect to $W$ such as

$$
r\left(x_{i} \mid W\right)
$$

By definition, we know that $\left|x_{i}-x_{j}\right| \leq \Delta$, and thus $r\left(x_{j} \mid W\right)=(\Delta, 0)$. Likewise, we know that $f(d) \leq f_{\max }(d)<\infty$, such that $|g-x|:=0<\gamma \Delta \leq N \Delta$ for $1 \leq \gamma \leq \infty$ so $r(g \mid W)=(\gamma \Delta, 0)$.

We now analyze the connectivity criteria for different types of configurations given the utility updating time-delays. 
For two random unmarked nodes $i, j \in \Lambda^{u}$, linked by $\theta_{u \backslash u}$, we have

$$
\dot{d}_{i j}^{2}=-2 N d_{i j}\left[d_{i j}-d_{k}\left(\varepsilon_{i}-\varepsilon_{j}\right)\right]
$$

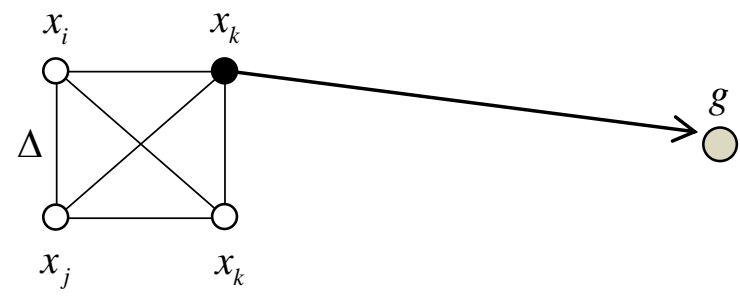

Fig. 2 Unmarked nodes

When $d_{i j} \rightarrow \Delta$, we have $\dot{d}_{i j}^{2} \leq 0$ if $d_{i j} \geq d_{k}\left(\varepsilon_{i}-\varepsilon_{j}\right)$. If the distance between an unmarked node and the barycenter weighted by the net utility time-delay is less than or equal to the distance between the nodes, unmarked nodes remain connected. Furthermore, by the law of cosines, we have $d_{k} \equiv d_{i, j} \cos (\alpha)$, so the condition resumes to $\frac{d_{i j}}{d_{i, j} \cos (\alpha)} \geq \varepsilon_{i}-\varepsilon_{j}$. The metric yields $\quad r\left(x_{j} \mid W\right)=(\Delta, 0) \quad$ and $\quad r\left(\sum \frac{x_{k}}{N} \mid W\right)=(\gamma \Delta \cos (\alpha), 0), \quad$ thus $\quad \frac{\Delta}{\gamma \Delta \cos (\alpha)} \geq \varepsilon_{i}-\varepsilon_{j} \quad$ or $\frac{1}{\gamma \cos (\alpha)} \geq \varepsilon_{i}-\varepsilon_{j}$. We thus fall on the secant function such that $\frac{\sec (\alpha)}{\gamma} \geq \varepsilon_{i}-\varepsilon_{j}$. Assuming $0<\alpha \leq \frac{\pi}{2}$ gives $\frac{1}{\gamma}<\sec (\alpha) \leq \frac{\tilde{\omega}}{\gamma}$ thus $\frac{1}{\gamma}<\varepsilon_{i}-\varepsilon_{j}$. In parallel, $\gamma \geq 1$ by assumption, so $\varepsilon_{i}>\varepsilon_{j}$. If this strict inequality is verified, unmarked nodes will remain connected as $t \rightarrow \infty$.

Rule 1: While being taken to the objective position, unmarked nodes must not be driven away beyond their utility domain by the utility updating time-delays. Otherwise, they disconnect. A necessary and sufficient condition for connectivity is asynchronous time-delays. 
For two random target nodes $i, j \in \Lambda^{t}$, linked by $\theta_{t \mid t}$, and $F\left(x_{i}, g\right) \neq 0$ and $F\left(x_{j}, g\right) \neq 0$, we have

$$
\dot{d}_{i j}^{2}=-2 N d_{i j}\left[d_{i j}-d_{k}\left(\varepsilon_{i}-\varepsilon_{j}\right)\right]-2 d_{i j}\left[f\left(d_{i}\right) d_{i}-f\left(d_{j}\right) d_{j}\right]
$$

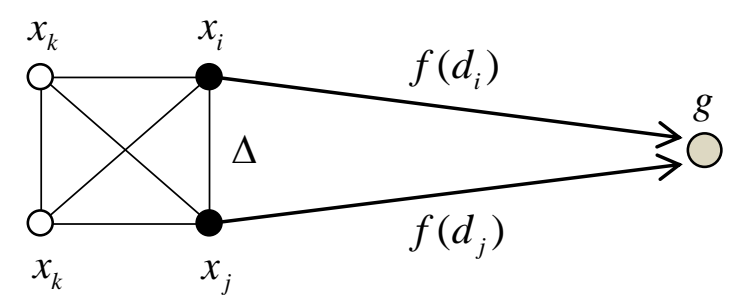

Fig. 2 Target nodes and the objective position

We have $\dot{d}_{i j}^{2} \leq 0$ when two conditions are met. The first is $d_{i j} \geq d_{k}\left(\varepsilon_{i}-\varepsilon_{j}\right)$ which occurs for $\varepsilon_{i}>\varepsilon_{j}$ (see Rule 1). The second condition implies that $f\left(d_{i}\right) d_{i}-f\left(d_{j}\right) d_{j} \geq 0$. If $K \subseteq \Omega$ is a convex subset of a Banach space and $f(\cdot)$ a real-valued function that is Fréchet differentiable, $\mathrm{d} f$ is an increasing monotone operator such that $\left[\mathrm{d} f\left(d_{i}\right) d_{i}-\mathrm{d} f\left(d_{j}\right) d_{j}\right] \geq 0$ $\forall i, j \in \Lambda^{t}$. The metric implies that $f\left(\gamma_{i} \Delta\right) \gamma_{i} \Delta-f\left(\gamma_{j} \Delta\right) \gamma_{j} \Delta \geq 0$ or $\gamma_{i} \Delta \geq \gamma_{j} \Delta$. Thereby, if the difference in distances towards the objective position is positive or null, target nodes remain within the convex hull.

Rule 2a: The effort engaged to transpose target nodes to the objective position and the respective utility updating time-delays it entails must not break their utility domain. Otherwise, they disconnect. Necessary and sufficient conditions for connectivity are increasing monotone objective function and asynchronous time-delays. 
For two random target nodes $i, j \in \Lambda^{t}$, linked by $\theta_{t \mid t}$, and $F\left(x_{i}, g\right)=0$ and $F\left(x_{j}, g\right)=0$, and for $d_{i j}=u_{i j}$, we have

$$
\dot{d}_{i j}^{2}=-2 N d_{i j}\left[d_{i j}-d_{k}\left(\varepsilon_{i}-\varepsilon_{j}\right)\right]
$$

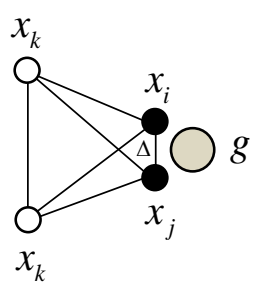

Fig. 3 Target nodes at the objective position

When $d_{i j} \rightarrow \Delta$, we have $\dot{d}_{i j}^{2} \leq 0$ if $d_{i j} \geq d_{k}\left(\varepsilon_{i}-\varepsilon_{j}\right)$ which is verified for $\varepsilon_{i}>\varepsilon_{j}$.

Rule 2b: While being upheld at the objective position, target nodes must not be driven away beyond their utility domain by the utility updating time-delays. Otherwise, they disconnect. A necessary and sufficient condition for connectivity is asynchronous time-delays.

For two random target nodes $i, j \in \Lambda^{t}$, linked by $\theta_{t \mid t}$, and $F_{i}\left(x_{i}, g_{i}\right) \neq 0$ and $F_{j}\left(x_{j}, g_{j}\right) \neq 0$, where $g_{i} \neq g_{j}$ or $f_{i}\left(d_{i}\right) \neq f_{j}\left(d_{j}\right)$, we have

$$
\dot{d}_{i j}^{2}=-2 N d_{i j}\left[d_{i j}-d_{k}\left(\varepsilon_{i}-\varepsilon_{j}\right)\right]-2 d_{i j}\left[f_{i}\left(d_{i}\right) d_{i}-f_{j}\left(d_{j}\right) d_{j}\right]
$$




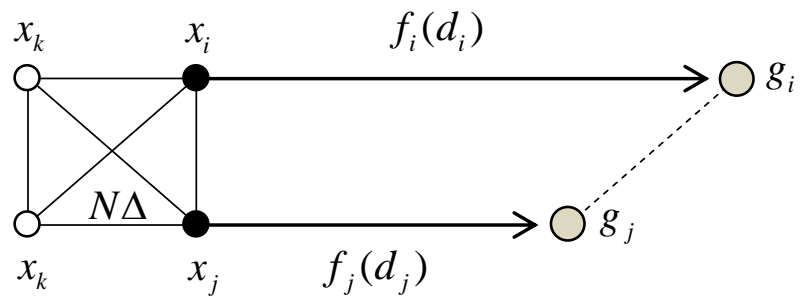

Fig. 4 Target nodes and the objective positions

We have $\dot{d}_{i j}^{2} \leq 0$ when two conditions are met. The first condition is $d_{i j} \geq d_{k}\left(\varepsilon_{i}-\varepsilon_{j}\right)$ which is verified for $\varepsilon_{i}>\varepsilon_{j}$. The second condition implies that $f_{i}\left(d_{i}\right) d_{i}-f_{j}\left(d_{j}\right) d_{j} \geq 0$ or $\frac{f_{i}\left(d_{i}\right)}{f_{j}\left(d_{j}\right)} \geq \frac{d_{j}}{d_{i}}$. Since $f_{\max }(d) \geq f(d)$ and $f_{\max }(d) \leq N u$, the precedent produces $\frac{d_{j}}{d_{i}} \leq \frac{N u}{N u}$. The metric gives $\frac{N \Delta}{N \Delta} \geq \frac{\gamma_{j} \Delta}{\gamma_{i} \Delta}$ so $\frac{\gamma_{j} \Delta}{\gamma_{i} \Delta} \leq 1$ and $\gamma_{i} \Delta \geq \gamma_{j} \Delta$. Once again, if the difference in distances towards separate objective positions is positive or null, target nodes remain within the convex hull.

Rule 2c: The efforts engaged to transpose target nodes to the separate objective positions and the respective utility updating time-delays they entail must not break their utility domain. Otherwise, they disconnect. Necessary and sufficient conditions for connectivity are respective maximum pressures on target nodes no greater than the network utility domain and asynchronous time-delays.

For two random target nodes $i, j \in \Lambda^{t}$, linked by $\theta_{t \mid t}$, for $F_{i}\left(x_{i}, g_{i}\right)=0$ and $F_{j}\left(x_{j}, g_{j}\right)=0$, where $g_{i} \neq g_{j}$ or $f_{i}\left(d_{i}\right) \neq f_{j}\left(d_{j}\right)$, we have

$$
\dot{d}_{i j}^{2}=-2 N d_{i j}\left[d_{i j}-d_{k}\left(\varepsilon_{i}-\varepsilon_{j}\right)\right]
$$




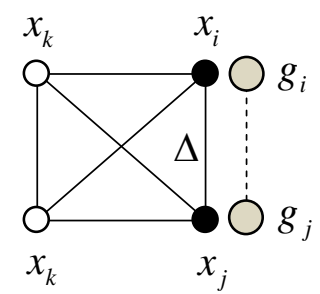

Fig. 5 Target nodes at the objective position

When $d_{i j} \rightarrow \Delta$, we have $\dot{d}_{i j}^{2} \leq 0$ if $d_{i j} \geq d_{k}\left(\varepsilon_{i}-\varepsilon_{j}\right)$ which is verified for $\varepsilon_{i}>\varepsilon_{j}$.

Rule 2d: While being upheld at the objective position, target nodes must not be driven away beyond their utility domain by the utility updating time-delays. Otherwise, they disconnect. A necessary and sufficient condition for connectivity is asynchronous time-delays.

For two random nodes $i \in \Lambda^{u}$ and $j \in \Lambda^{t}$, linked by $\theta_{t \backslash u}$, and for $F\left(x_{j}, g\right) \neq 0$, we have

$$
\dot{d}_{i j}^{2}=-2 N d_{i j}\left[d_{i j}-d_{k}\left(\varepsilon_{i}-\varepsilon_{j}\right)\right]-2 d_{i j} f\left(d_{j}\right) d_{j}
$$

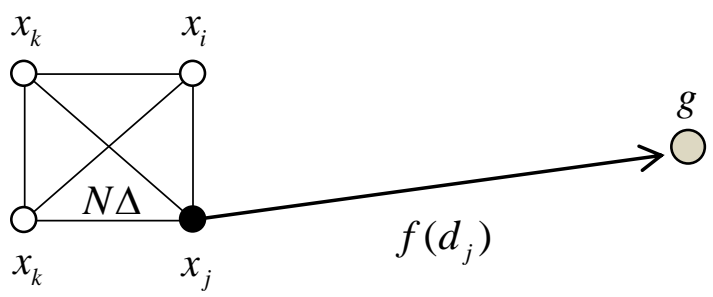

Fig. 6 Unmarked node and target node

By assumption, $f(d) \geq 0$, so $\dot{d}_{i j}^{2} \leq 0$ as long as $\varepsilon_{i}>\varepsilon_{j}$. 
Rule 3a: The effort engaged to transpose the target node to the objective position and the respective utility updating time-delays it entails must not break the utility domain between target and unmarked nodes. Otherwise, they disconnect. Necessary and sufficient conditions for connectivity are positive objective function and asynchronous time-delays.

For two random nodes $i \in \Lambda^{u}$ and $j \in \Lambda^{t}$ linked by $\theta_{t \mid u}$, and for $F\left(x_{j}, g\right)=0$, we know

$$
\dot{d}_{i j}^{2}=-2 N d_{i j}\left[d_{i j}-d_{k}\left(\varepsilon_{i}-\varepsilon_{j}\right)\right]
$$

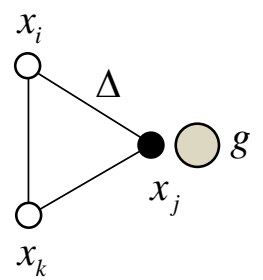

Fig. 7 Unmarked node and target node at the objective position

When $d_{i j} \rightarrow \Delta$, we have $\dot{d}_{i j}^{2} \leq 0$ if $d_{i j} \geq d_{k}\left(\varepsilon_{i}-\varepsilon_{j}\right)$ which is verified for $\varepsilon_{i}>\varepsilon_{j}$.

Rule 3b: While being upheld at the objective position, unmarked and target nodes must not be driven away beyond their utility domain by the utility updating time-delays. Otherwise, they disconnect. A necessary and sufficient condition for connectivity is asynchronous time-delays.

Overall, we can see that the time-delay that can be tolerated by a connected network applying a consensus protocol depends on rather simple management constraints such as asynchronous time-delays and standard criteria towards the objective transposition function. 


\section{Conclusion}

This paper aimed at formalizing the criteria for a sustainable ecosystem management. We first proved the existence of a subset of target nodes identified by an anthropogenic controller and introduced the Coherence Principle in order to avoid the paradox of multiple attributes over nodes. We then modeled the impossibility for a network to translate its barycenter to the objective position via Impossibility Theorem.

This result enabled to reveal the minimal Sustainability Criterion which ensures that the network elements of the ecosystem remain connected while being conveyed to the objective position issued from the environmental policy. The criterion guarantees that the ecosystem maintains its self-organizing structural complexity or integrity and preserves its utility domain. Our approach meets that of Smith et al. (2009) who assert that optimal policies must be determined over the subsets of connected systems. At last, we identified three management rules to ensure the maintenance of connectivity in time, given the property of the objective transposition function, the nature of connections and utility updating time-delays between the nodes.

\section{References}

Albers, H. (1996), "Modeling Ecological Constraints on Tropical Forest Management: Spatial Interdependence, Irreversibility, and Uncertainty", Journal of Environmental Economics and Management, 30: 73-94.

Cantwell, M. and Forman, R. (1993), "Landscape Graphs: Ecological Modeling with Graph Theory to Detect Configurations Common to Diverse Landscapes", Landscape Ecology, 8: 239-255.

Callicott, J. (1993), "A Brief History of American Conservation Philosophy", in W. Wallace Covington and Leonard F. Debano, eds., Sustainable Ecological Systems: Implementing an Ecological Approach to Land Management, Rocky Mountain Forest and Range Experiment Station, Fort Collins, CO (USA).

Chomette, T. (2010), "Jeux sur les Graphes et Théorie de Ramsey", CultureMATH, available at http://www.math.ens.fr/culturemath/maths/pdf/jeux/ramsey.pdf

Christensen, N., Bartuska, A., Brown, J., Carpenter, S., D’Antonio, C., Francis, R., Franklin, J., MacMahon, J., Noss, R., Parsons, D., Peterson, C., Turner, M. and Woodmansee, 
R. (1996), "The Report of the Ecological Society of America Committee on the Scientific Basis for Ecosystem Management", Ecological Applications, 6: 665-691.

Clarke, P. and Jupiter, S. (2010), "Principles and Practice of Ecosystem-Based Management: A Guide for Conservation Practitioners in the Tropical Western Pacific", Wildlife Conservation Society, Suva, Fiji.

Connell, J. and Sousa, W. (1983), "On the Evidence Needed to Judge Ecological Stability or Persistence", American Naturalist, 121: 789-824.

Constanza, R., Norton, B. and Haskell, B. (1992), "Ecosystem Health: New Goals for Environmental Management", Island Press, Washington, DC.

Dattorro, J. (2005), "Convex Optimization \& Euclidean Distance Geometry", Meboo Publishing, Palo Alto, California, USA.

Fath, B. (2004), "Distributed Control in Ecological Networks", Ecological Modelling, 179: 235-245.

Fath, B. and Grant, W. (2007), "Ecosystems as Evolutionary Complex Systems: Network Analysis of Fitness Models", Environmental Modelling and Software, 22: 693-700.

Fath, B. and Patten, B. (1998), "Network Synergism: Emergence of Positive Relations in Ecological Systems ", Ecological Modelling, 107: 127-143.

Fisher, A., Hanemann, M. and Keeler, A. (1991), "Integrating Fishery and Water Resource Management: A Biological Model of a California Salmon Fishery", Journal of Environmental Economics and Management, 20: 234-261.

Gazi, V. and Passino, K. (2004), "A class of Attractions/Repulsion Functions for Stable Swarm Aggregations", International Journal of Control, 77: 1567-1579.

Graham, R., Rothschild, B., Spencer, J. (1990), "Ramsey Theory", New York: John Wiley and Sons.

Gustavi, T., Dimarogonas, D., Egerstedt, M. and Hu, X. (2010), "Sufficient Conditions for Connectivity Maintenance and Rendezvous in Leader-Follower Networks", Automatica, 46: 133-139.

Jensen, M., Reynolds, K., Langner, U. and Hart, M. (2009), "Application of Logic and Decision Models in Sustainable Ecosystem Management", Proceedings of the 42nd Hawaii International Conference on Systems Sciences, Waikoloa, Hawaii. 5-8 January 2009.

Ji, M., Ferrari-Trecate, G., Egerstedt, M. and Buffa, A. (2008), "Containment Control in Mobile Networks", IEEE Transactions of Automatic Control, 53: 1972-1975.

Jordan, F. and Scheuring, I. (2004), "Network Ecology: Topological Constraints on Ecosystem Dynamics", Physics of Life Reviews, 1: 139-172.

Lanzen, M. (2007), "Structural Path Analysis of Ecosystem Networks", Ecological Modelling, 200: 334-342.

Lee, K. (1993), "Compass and Gyroscope: Integrating Science and Politics for the Environment", Island Press, Washington, DC.

Mesbahi, M. and Egerstedt, M. (2010), "Graph Theoretic Methods in Multiagent Networks ", Princeton Series in Applied Mathematics, Princeton University Press, Princeton, NJ.

Olfati Saber, R. and Murray, R. (2004), "Consensus Problems in Networks of Agents with Switching Topology and Time-Delays", IEEE Transactions on Automatic Control, 49: $1520-1533$.

Patten, B. (1981), "Environs: The Superniches of Ecosystems", American Zoologist, 21: 845852.

Patten, B. (2010), "Natural Ecosystem Design and Control Imperatives for Sustainable Ecosystem Services", Ecological Complexity, 7: 282-291.

Payton, I., Fenner, M., Lee, W. (2002), "Keystone Species: The Concept and Its Relevance for Conservation Management in New Zeland", Science for Conservation, 203: 5-29. 
Peterson, C. (1993), "Improvement of Environmental Impact Analysis by Application of Principles Derived from Manipulative Ecology: Lessons from Coastal Marine Case Histories", Australian Journal of Ecology, 18: 21-52.

Pichai, V., Sezer, M., Siljak, D. (1981), "Vulnerability of Dynamic Systems", International Journal of Control, 34: 1049-1060.

Power, M., Tilman, D., Estes, J., Menge, B., Bond, W., Mills, L., Daily, G., Castilla, J., Lubchenco, J. and Paine, R. (1996), "Challenges in the Quest for Keystones", Bioscience, 46: 609-620.

Rahmani, A., Ji, M., Mesbahi, M. and Egerstedt, M. (2009), "Controllability of Multi-Agent Systems from a Graph-Theoretic Perspective", SIAM Journal on Control and Optimization, 48: 162-186.

Reynolds, K. (2005), "Integrated Decision Support for Sustainable Forest Management in the United States: Fact of Fiction?", Computers and Electronics in Agriculture, 49: 6-23.

Siljak, D. (1991), "Decentralized Control of Complex Systems", Academic Press, Boston, MA.

Smith, M., Sanchirico, J. and Wilen, J. (2009), "The Economics of Spatial-Dynamic Processes: Applications to Renewable Resources", Journal of Environmental Economics and Management, 57: 104-121.

Spanos, D., Olfati-Saber, R., and Murray, R. (2005), "Dynamic Consensus on Mobile Networks", International Federation of Automatic Control World Congress.

USDA Forest Service (2008), "National Forest System Land Management Planning; Final Rule", Report 36 CFR Part 219 available at http://www.fs.fed.us.

\section{Appendix}

\section{- Proof of the Identification Theorem}

We have to prove the theorem for two types and then extend it to more than two types. That is, for every interaction in $\Gamma_{n}$ in type $\theta_{i}$, where $i=1,2$, there exists integer $n$ such that $\Lambda_{\theta_{1}} \subseteq \Gamma_{n}$ or $\Lambda_{\theta_{2}} \subseteq \Gamma_{n}$, and then extend the proof to $i>2$.

We first prove that the theorem holds for two types of interactions, thus $r=2$.

Let $n \rightarrow\left(\theta_{1}, \theta_{2}\right)$ be that integer $n$ is true for $\left(\theta_{1}, \theta_{2}\right)$. This means that for every interaction in $\Gamma_{n}$ in $\theta_{1}$ or $\theta_{2}$, we have subgraph $\Lambda_{\theta_{1}}$ or $\Lambda_{\theta_{2}}$. By induction, we show that for $\left(\theta_{1}, \theta_{2}\right)$ there 
exists $n$ such that $n \rightarrow\left(\theta_{1}, \theta_{2}\right)$, that is, the whole graph interacts via the two types of interactions.

Let $\left(\theta_{1}, \theta_{2}\right)$ such that $\theta_{1}+\theta_{2}=\theta$. Assume the result is true for some $\left(\varphi_{1}, \varphi_{2}\right)$ with $\varphi_{1}+\varphi_{2}<\theta$ and thus for $n_{1}$ and $n_{2}$ such that $n \rightarrow\left(\theta_{1}-1, \theta_{2}\right)$ and $n \rightarrow\left(\theta_{1}, \theta_{2}-1\right)$. These integers exist only because of the induction method. We show that $n=n_{1}+n_{2}$ verifies $n \rightarrow\left(\theta_{1}, \theta_{2}\right)$.

Consider a node $s_{1}$ selected from $\Gamma_{n}$. Define subsets $\Lambda^{\theta_{1}}$ and $\Lambda^{\theta_{2}}$ of all remaining nodes, such that for all $s_{n-1} \neq s_{n}$, we have $s_{n-1} \in \Lambda^{\theta_{1}}$ if and only if the interaction $\left(s_{1}, s_{n-1}\right)_{\theta_{i}}$ is of type $\theta_{i}$, where $i=1,2$.

Subsets $\Lambda^{\theta_{1}}$ and $\Lambda^{\theta_{2}}$ constitute a partition of the set of the $n-1$ remaining nodes. Respective subgraphs $\Lambda_{\theta_{1}}$ and $\Lambda_{\theta_{2}}$ are obtained from nodes of these subsets. We have $\left|\Lambda_{\theta_{1}}\right| \geq n_{1}$ and $\left|\Lambda_{\theta_{2}}\right| \geq n_{2}$

By assumption, we know that $n_{1} \rightarrow\left(\theta_{1}-1, \theta_{2}\right)$. Hence, $\Lambda_{\theta_{1}} \supseteq \Lambda_{\theta_{1}-1}$ or $\Lambda_{\theta_{1}} \supseteq \Lambda_{\theta_{2}}$. In consequence, we have $\Lambda_{\theta_{2}} \subseteq \Gamma_{n}$.

We add the node selected at the beginning, which is linked to $\Lambda_{\theta_{1}}$ and thus to $\Lambda_{\theta_{1}-1}$. We now have a complete graph of $\theta_{1}-1+1=\theta_{1}$, in which all interactions are of the same type. By symmetry, we have the same case for $\left|\Lambda_{\theta_{2}}\right| \geq n_{2}$. By induction, we conclude that for $\left(\theta_{1}, \theta_{2}\right)$ there exists $n$ such that $n \rightarrow\left(\theta_{1}, \theta_{2}\right)$. 
Let us now consider the case with more than two types. In this case, $r=3$.

The result is immediate by induction. Assume the result holds for $\theta_{r}$ types of interactions. Let $n$ be integer such that $n \rightarrow(k)_{\theta_{r}}$, and $m$ be integer such that $m \rightarrow(n)_{\theta_{2}}$. Then integer $m$ verifies $m \rightarrow(k)_{\theta_{r}+1}$.

For every interaction in $\Lambda_{m}$, we already have an interaction of one of two types: the interaction is of type $\theta_{1}$ if and only if it was of type $\theta_{r}$ in the previous interaction. By induction, it means that the interaction of type $\theta_{2}$ was of the type $\theta_{r+1}$ in the previous interaction.

The theorem being proved for two types, we know that $\Lambda_{m} \supseteq \Lambda_{n}$. If $\Lambda_{n} \equiv \Lambda_{\theta_{2}}$, we have $\Lambda_{m} \supseteq \Lambda_{\theta_{2}}$ and consequently $\Lambda_{m} \supseteq \Lambda_{\theta_{1}}^{k}$ as $n \geq k$. Since $n \rightarrow(k)_{\theta_{r}}$, we have $\Lambda_{m} \supseteq \Lambda_{\theta_{r}}^{k}$. This ends the proof.

\section{- Sustainability Criterion}

$$
\begin{aligned}
\dot{x} & =(x-g)^{T}(x-g) \\
& =\left[x^{T} g^{T}\right]\left[\begin{array}{cc}
I & -I \\
-I & I
\end{array}\right]\left[\begin{array}{l}
x \\
g
\end{array}\right] \\
& =-\left[\left[x_{1}, \ldots, x_{N}\right]^{T}\left(L \otimes I_{2}\right)+\left[F_{1}(x, g), \ldots, F_{n}(x, g)\right]^{T}\right] \\
\dot{V} & =(x-g)^{T}(\dot{x}-\dot{g}) \\
& =-\left[(x-g)^{T}\left(L \otimes I_{2}\right)(x-g)\right]-\sum_{i \in \Lambda^{t}} F\left(x_{i}, g\right)
\end{aligned}
$$

